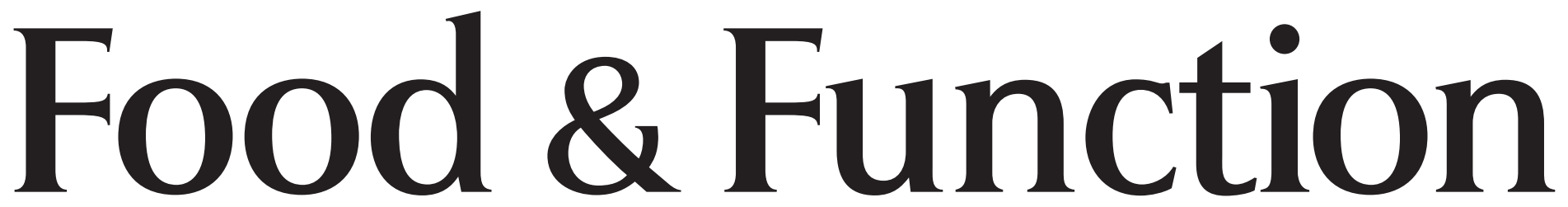

Linking the chemistry and physics of food with health and nutrition

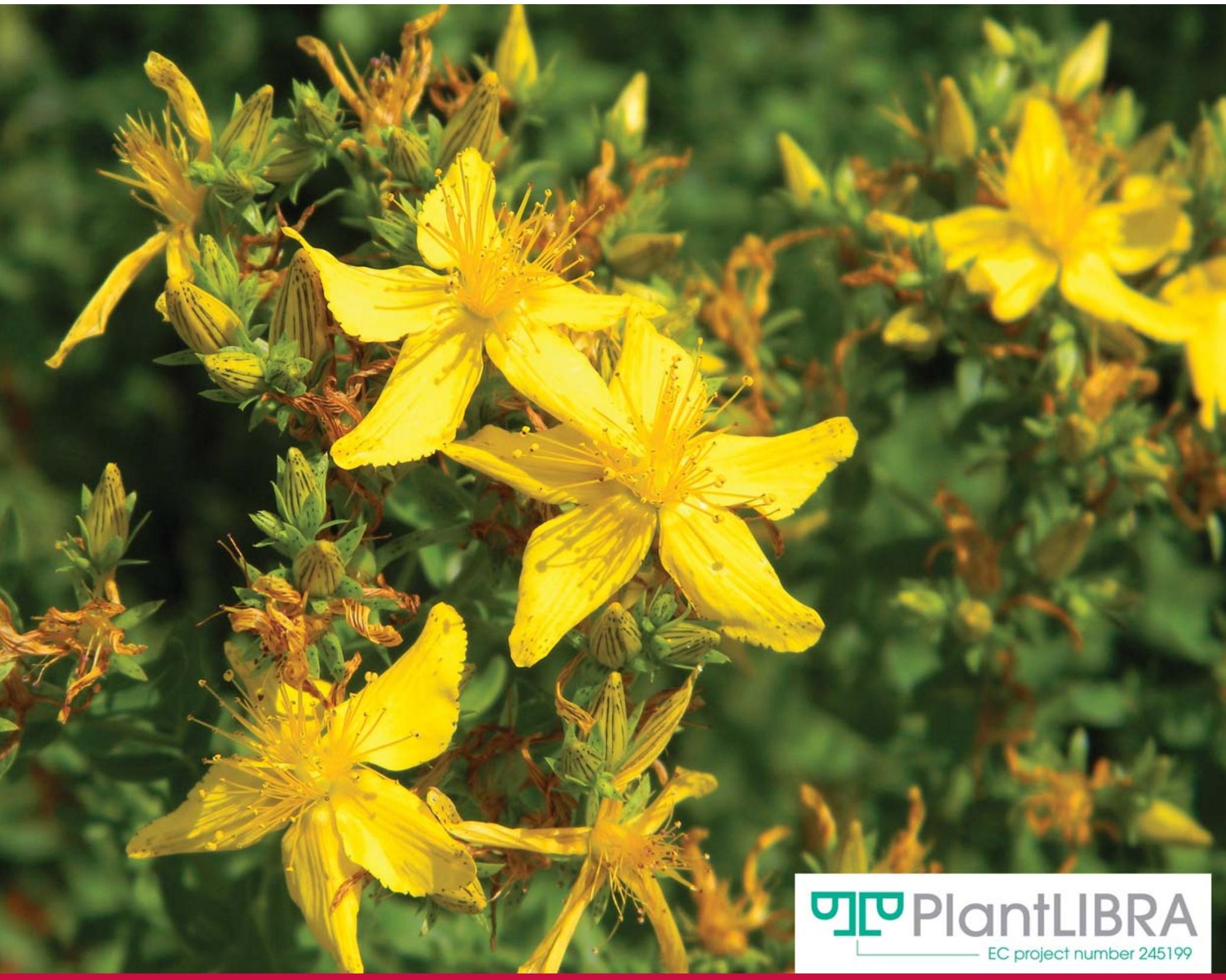

Themed issue: Plant food supplements

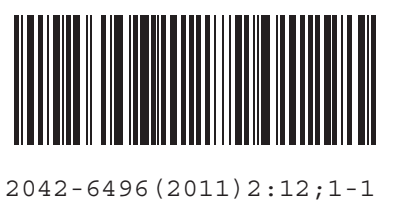




\title{
The PlantLIBRA Project: how we intend to innovate the science of botanicals $\dagger+$
}

\author{
Luca Bucchini, ${ }^{a}$ Alejandro Rodarte ${ }^{a}$ and Patrizia Restani ${ }^{* b}$ \\ Received 21st July 2011, Accepted 17th November 2011 \\ DOI: 10.1039/c1fo10150a
}

\begin{abstract}
The main aim of the EC-financed R\&D project PlantLIBRA (PLANT food supplements: Levels of Intake, Benefit and Risk Assessment) is to foster the safe use of food supplements containing plants or botanical preparations, by enabling science-based decision making by regulators and stakeholders. To make informed decisions, competent authorities and industry need more accessible and quality-assured information, as well as better tools (e.g., databases) and procedures for safety and benefit assessments, supported by broadly accepted methodologies. Consequently, PlantLIBRA is working to develop, validate and disseminate data and methodologies for risk and benefit assessment of plant food supplements, and to implement sustainable international cooperation. International cooperation will help ensure the quality of botanicals imported in the EU. Moreover, the project will provide data on intake by conducting a harmonized consumption survey. Existing composition and safety data will be collated into a meta-database. New analytical data and methods will be investigated and validated. The consortium is working closely with competent authorities and stakeholders.
\end{abstract}

\section{Introduction}

Worldwide, there is a growing demand for high-quality, safe, health-promoting or disease-risk reducing foods, including food supplements.

Plant Food Supplements (PFS) have shown a high acceptance by consumers ${ }^{1}$ due to potential/advertised advantages like efficacy, safety, and relatively low costs. ${ }^{2}$ They have also been used for their health-promoting effects for a long time. PFS contain plants or herbal extracts and are defined in the EC Directive 2002/46 on general provisions for food supplements, ${ }^{3}$ as foodstuffs whose purpose is to supplement the normal diet and which are concentrated sources of nutrients or other substances with a nutritional or physiological effect, alone or combined, marketed in dose form, such as capsules, pastilles, tablets, pills and other similar forms, sachets of powder, ampoules of liquids, drop dispensing bottles, and other similar forms of liquids and powders designed to be dispensed in measured small unit quantities.

${ }^{a}$ Hylobates Consulting srl, Via Tarsia 61, Rome, Italy. E-mail. lucabucchini@hylobates.it; alejandrorodarte@hylobates.it; Fax: +39 0698309396 28; Tel: +3906989396 30

${ }^{b}$ Dept. Pharmacological Sciences, Università degli Studi di Milano, Via Balzaretti 9, 20133 Milano, Italy. E-mail: patrizia.restani@unimi.it; Fax: +39025031 8284; Tel: $+390250318371 / 350$

$\dagger$ This paper forms part of the themed issue on Plant Food Supplements: regulatory, scientific and technical issues concerning safety, quality and efficacy.

\$ The content of this summary is based on the PlantLIBRA project's statement of work and the tasks achieved till the point of writing of this summary.
The recent discussions within Europe and elsewhere have identified several knowledge gaps in the way these products are tested and evaluated in terms of efficacy and safety. Furthermore, a shared approach to evaluate the benefits and safety of PFS across the EU would be helpful for the scientific assessments and contribute to international harmonization.

In terms of safety, EFSA, the European Food Safety Authority, has initiated work in this area and has elaborated a guidance for the safety assessment of botanicals and botanical preparations. ${ }^{4}$ In their assessment, EFSA has also identified bottlenecks that ought to be addressed. More generally, with the overall purpose of harmonising and monitoring global food quality and safety, several European Commission-funded research projects ${ }^{5}$ have established methodologies and developed tools, predominantly for foods, that could, but have not been yet applied to PFS. These projects include:

- EuroFir (European Food Information Resource),

- MoniQa (Monitoring and Quality Assurance in the Food Supply Chain),

- Beneris (Benefit-Risk Assessment for Food),

- Qalibra (Quality of Life - Integrated Benefit and Risk Analysis), and

- Eurreca (European micronutrient Recommendations Aligned).

In order to better protect consumers, a new integrated approach was needed for plant food supplements. In this context and to address this situation, the PlantLIBRA (acronym of PLANT food supplements: Levels of Intake, Benefit and Risk Assessment) project was created in response to the 7th EU Framework Programme call for proposals titled: Risk-benefit 
assessment of food supplements. This topic was within the European Commission's funding scheme Collaborative Project (large scale integrating project) for Specific International Cooperation Actions (SICA). After being positively evaluated, the project started in June 2010 and will end in May 2014.

\section{Safe use of plant food supplements with science- based decision making}

The overall goal of PlantLIBRA is to improve the scientific knowledge base of PFS to better assess their risks and benefits, and ultimately ensure a safer use of PFS by consumers. This requires consensus on a framework backed up by adequate scientific data. The framework should be practical, use-specific and accepted by decision-makers.

In this context, PlantLIBRA will contribute by:

(1) Expanding and generating knowledge through systematic reviews, from published or previously inaccessible datasets, and through new studies on benefits, risks and new analytical findings. This will be complemented with research on plants used for food supplements, on their phytochemical composition, and the analytical methods for beneficial or toxic components.

Subsequently, this body of knowledge will be transferred to an accessible meta-database. The database will be easily searchable with retrievable data on chemical composition, botanical information, bioactivity and toxicity data. It will be developed to serve a variety of needs of the PlantLIBRA project, of other end users and of stakeholders.

(2) Providing regulators across the EU with an enhanced decision-making framework to evaluate and assess benefits and risks, integrating and advancing existing tools (e.g., EFSA and other international guidelines). This will enable and help increase science-based decision making by regulatory authorities and players of the PFS supply chain in the EU and in exporting countries.

(3) Contributing to a better understanding of consumer behaviour through the first pan-European PFS intake survey. The results of the survey, further studies on consumer behaviour and the direct interaction with regulatory authorities, can provide useful information to stakeholders and regulators to enhance the safe use of food supplements by consumers.

\section{Key activities of the PlantLIBRA project}

The project activities are divided in work packages which address the specific goals of PlantLIBRA. These comprise intake, quality and composition, efficacy, consumer perceptions, adverse effects and safety, risk-benefit assessment, and policy implications (see Fig. 1).

\subsection{Estimation of plant food supplements intake}

Consumption of PFS in key European countries (Finland, Germany, Italy, Romania, Spain and United Kingdom) will be estimated. For this purpose, data on market trends, places of purchase and determinants of consumption and of usage have been collected from various sources: scientific literature, grey literature, and questionnaires to key informants from industry, professional associations and public institutions. This was followed by the start of an ongoing European PFS consumption survey in the above-mentioned countries, the results of which will provide data on PFS consumption patterns, intake related factors (e.g. lifestyle habits such as smoking, alcohol and use of other medications, socioeconomic status, health status, etc.), adherence to product usage recommendations, and determinants of usage. The research conducted will also allow the harmonisation of PFS terminology and their classification.

To obtain reliable results and considering the significant differences within the European market of PFS, the questionnaires have been carefully formulated to reflect the variability of the products and distinguish between PFS and herbal medicines. For this purpose, interviewers have been trained regarding concepts, definitions, and product differences. The data collected from the survey is verified accordingly as well.

\subsection{Investigation on botanical ingredients and plant food supplements}

The objective is to provide new experimental data and consensus on the best methods with regards to:

(1) identification of plant material;

(2) analysis of bioactive constituents with positive or negative health effects;

(3) detection of environmental contaminants or residues from agricultural practice;

(4) biological markers useful to evaluate exposure, physiological activity, adverse effects and/or misidentification of plants.

Information gaps will also be identified and the generated outputs will expand the body of knowledge in the meta-database.

Priority methods for the determination of bioactive compounds, contaminants, pesticide residues and biomarkers occurring in PFS have been identified, and will be evaluated and validated. To this end, priority lists of compound classes and plants according to relevance, toxicity, benefits, risks and other criteria have been drafted.

This approach includes the development and testing of methods for the detection of compounds useful for identification and to test for physiological/toxicological activity. Additionally, an international network of laboratories will be organized with proven expertise in analytical assays for PFS and botanicals. The laboratories selected for the network will have to fulfil specific quality criteria and excel in technical and analytical competence, and possess state-of-the-art methodologies. The network aims to promote further investigation in the sector and provide authorities, and other stakeholders, with reference laboratories for quality and safety issues concerning PFS.

\subsection{Investigation on adverse effects to botanicals and plant food supplements}

The activities include:

(1) collection of well-documented adverse effects in humans to plants consumed as food or as ingredients of food supplements, with information and characterization of botanicals, their role in adverse effects or their misidentification (supported by data from European Poison Centres);

(2) identification and/or confirmation of biological markers of adverse effects; 


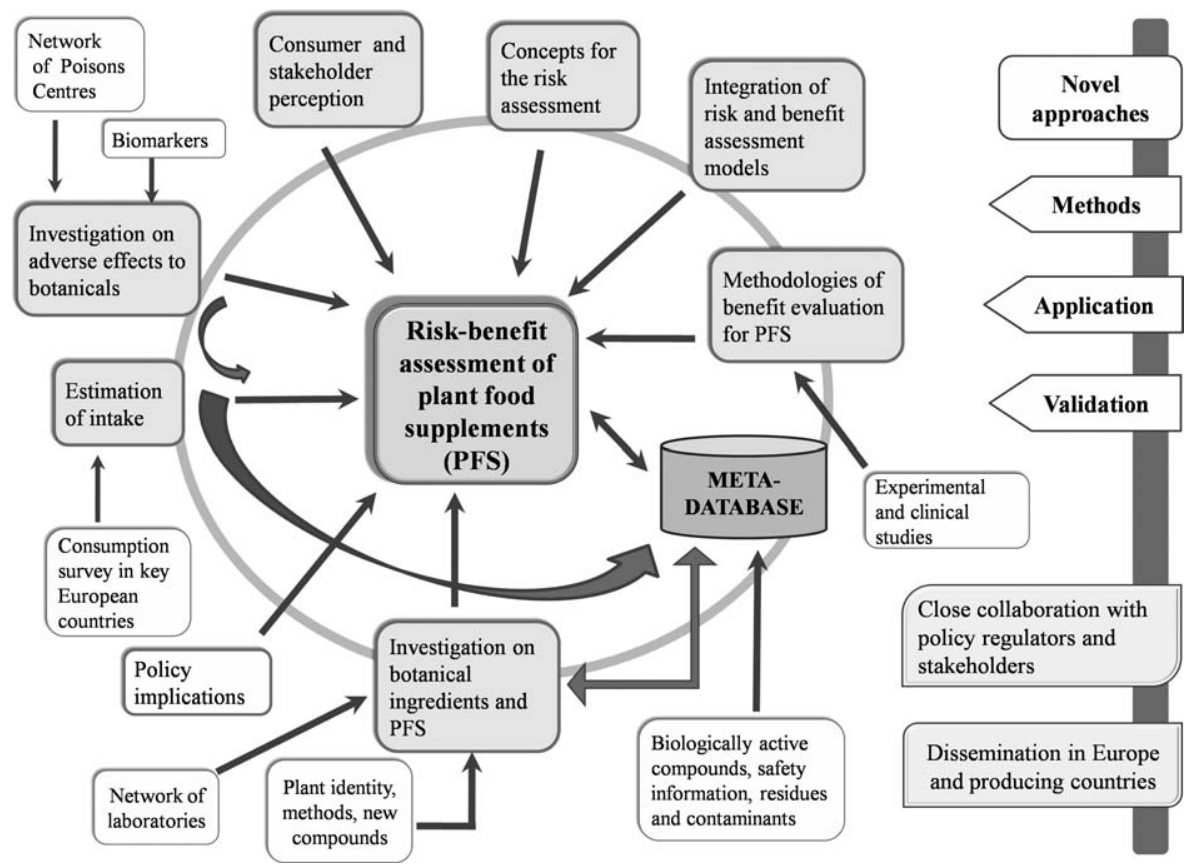

Fig. 1 Schematization of the work scope of the PlantLIBRA project for the assessment of plant food supplement (PFS) safety.

(3) assessment of source of misidentification of plants used as food or food supplements, and containing poisonous compounds;

(4) collection of data on known interactions between plant ingredients and nutrients, or plant ingredients and conventional drugs;

(5) collaboration with the existing international alert network (via the European Association of Poisons Centres and Clinical Toxicologists - EAPCCT) to improve the dissemination of data on adverse effects and poisoning due to botanicals.

\subsection{Meta-database of composition, biologically active compounds, safety information, residues and contaminants}

A fundamental output of PlantLIBRA is a database. It will address the needs of regulators and industry, who need to know more about plants used in supplements. In this sense, the PlantLIBRA meta-database combines databases on plant compounds, extracts, analytical methods, case-reports of adverse effects, literature on benefits and risks, alerts produced by authorities, and potential contaminants, into a single platform to enable risk and benefit assessments.

The meta-database (ePlantLIBRA) is based on existing databases: eBASIS (Bioactive Substances in Food Information System), developed by EuroFIR, and the MoniQA methods database.

The primary goal of this activity is to develop, test and apply a sustainable integrated meta-database of biologically active compounds found in plants used for manufacturing PFS. The meta-database (ePlantLIBRA) is based on existing databases: eBASIS (Bioactive Substances in Food Information System), developed by EuroFIR, and the MoniQA methods database.

The ePlantLIBRA database will provide the methodology and data, or pertinent links to other databases, to allow decision makers evaluating risks and benefits to retrieve comprehensive science-based information on plants and food supplements.

\subsection{Evaluation of the effects of plant food supplements}

The main goals are the identification, application and validation of methodologies to evaluate beneficial effects of consumption of priority PFS. To obtain these results, biomarkers of exposure specific for PFS which are commonly consumed will be reviewed and validated in a human study. It should be noted that biomarkers of exposure are relevant to both benefit and risk, since the effects of PFS are dependent on the dose. In this context, the benefits reachable by the consumption of food supplements could be defined as "the attainment of specific physiological objectives, such as reduction of risk factors for chronic diseases and the maintenance of the human homeostasis, which is the body's capability to physiologically regulate wellbeing and ensure stability and balance in response to changes in the external environment".

Short and simple human intervention studies with a single quantified dose of selected PFS will be carried out to determine further biomarkers of exposure in urine. Urine is, in most cases, the best source of biomarkers of exposure and is much less invasive than blood sampling.

A series of "best practice" recommendations for future human intervention studies on PFS will be developed, based on a systematic review of existing human intervention studies using PFS. Regarding the potential beneficial effects of PFS, it is critical to enable an early evaluation of their efficacy. This is measured by in vitro assays as an indication of activity in vivo. A panel of assays will be investigated to identify the most efficient for screening activities of different PFS.

\subsection{Concepts for the risk assessment of plant food supplements}

The objective is to provide an improved science-based model to evaluate the risks of consumption of food supplements containing or consisting of plants and/or botanical preparations used in the EU. 
A methodology for the risk assessment of botanicals and botanical preparations will be defined, based on existing models for foods, adapted to the requirements of PFS. The methodology to be developed and validated will focus on issues that, according to the present state of the art and recent guidelines and opinions from regulatory bodies, are the major bottlenecks in the risk assessment of PFS. For example, a quantitative approach, the Threshold of Toxicological Concern (TTC), has been evaluated for its potential for the risk assessment of PFS. Additionally, botanical ingredients have been selected, based on their genotoxic and/or carcinogenic potential, and assessed using the Margin of Exposure (MOE) approach.

The methodology will be tested using plant case studies and will address the major priorities that pertain to PFS, including consumption and exposure.

\subsection{Integration of risk and benefit assessment models, risk- benefit assessments and validation}

Benefit and risk assessment methodologies will be integrated in a general risk-benefit assessment model.

The main objectives are:

(1) to review science-based models to assess risks and benefits of foods and herbal medicines and their applicability for PFS containing plants and/or botanical preparations;

(2) to provide a flexible tool to screen risks, benefits and determine risk-benefit situations occurring in PFS;

(3) to identify used common currencies that are adjustable to the risks and benefits particular to PFS (like qualityadjusted life years (QALYs) or disability-adjusted life years (DALYs)).

The development, application and validation of the proposed approach will be evaluated through plant risk-benefit assessment case studies. Case studies will rely on the ePlantLIBRA database for data.

\subsection{Consumer and stakeholder perceptions of plant food supplements}

The project aims to deliver a better understanding of the perceptions of stakeholders and consumers towards PFS.

For this, it is necessary to map the consumer PFS information environment, to compare and contrast various perceptions, and to identify and model consumer responses to available information relating to PFS risk/benefits. It is assumed that consumers do not constitute a homogeneous category and several groups of consumers can be identified, including consumers and non-consumers of PFS.

To understand stakeholder and consumer perceptions of PFS, a mental models approach is proposed for this research:

(1) To obtain expert stakeholder beliefs towards PFS, factors influencing these beliefs, and how these relate to other aspects of diet and health. This will allow for mapping of areas of both agreement and disagreement between experts;

(2) To draw out lay consumer mental models to assess the nature and ways in which people think about different PFS;

(3) To assess the similarities and differences in the conceptualisations of PFS between expert stakeholders and lay consumers.

\section{General impact in the area of plant food supplements}

To facilitate development in the science of botanicals and deliver science-based guidance for the PFS sector, it is essential to clarify and report on the policy implications of the results of the project both at the EU and international level. Ultimately, this concerns both consumers and stakeholders and it aims for better consumer understanding and protection. These target groups includes citizens, the consumers of plant food supplements, and special interest groups, such as manufacturers, associations, consumer groups, pharmacists, retailers, and EU and international NGOs. The project also aims to promote innovation; an essential aspect of promoting innovation is through clarification and communication of project results.

Therefore the involvement of stakeholders, such as policy makers, industry and trade associations, and working with them in the form of advisory boards, provides the opportunity to address problems, and receive inputs on scientific bottlenecks and limitations in the sector. In September 2011, policy regulators from 19 European countries, China and the USA debated with PlantLIBRA researchers on outcomes and strategies to tackle priorities in the science of plant food supplements.

\subsection{International cooperation as a multiplier of project success}

European food supplement manufacturers operate in a globalized context, and therefore raw materials are frequently sourced from outside the European Union. Exporting countries face the same customs policy of the European Union and similar controls upon entry in the Union. As a consequence, an integrated approach is helpful for the whole EU and for exporting countries, and requires international cooperation.

The global impact of PlantLIBRA is accomplished through direct participation of four Extra-European institutions (Argentina, Brazil, China, and South Africa) in the project, by sharing their knowledge and expertise, through dissemination events and with mutual exchange.

\section{Conclusions}

The goals set by the PlantLIBRA consortium are certainly ambitious. The project consortium aims to address clear demands coming from different stakeholders in the field of PFS. Botanicals have a long tradition of use; their consumption and regulation are influenced by various historical, cultural and economic factors. In this context, the EC has acknowledged that for further advancements in the area of PFS, innovation and consensus is needed in policy and science to ensure the safety of consumers.

The PlantLIBRA consortium is working to address data, methodology and consensus gaps in cooperation with different stakeholders and decision makers in the PFS sector.

\section{Acknowledgements}

The writing of this summary was funded by the European Community's Seventh Framework Programme (FP7/2007-2013) under grant agreement $\mathrm{n}^{\circ} 245199$. It has been carried out within the PlantLIBRA project (website: www.plantlibra.eu). This 
report does not necessarily reflect the Commission's views or its future policy on this area.

\section{Notes and references}

1 Characteristics and perspectives of the market of food supplements containing substances other than vitamins and minerals, SEC(2008) 2976, EC, 2008, available online: http://ec.europa.eu/food/food/
labellingnutrition/supplements/documents/2008_2976_F_WD1_en. pdf.

2 E. Ernst, Herbal medicines put into context, Br. Med. J., 2003, 327, 881.

3 EC Directive 2002/46.

4 EFSA, EFSA J., 2009, 7(9), 280.

5 Related European Commission funded research projects: www.eurofir. net/, www.moniqa.org/, www.qalibra.eu/, www.beneris-qalibra.eu, www.eurreca.org/. 